\title{
Effect of the oxygen flow on the properties of ITO thin films deposited by ion beam assisted deposition (IBAD)
}

\author{
Li-Jian Meng $^{\mathrm{a}, \mathrm{b}, *}$, Jinsong Gao ${ }^{\mathrm{c}}$, R.A. Silva ${ }^{\mathrm{a}}$, Shigeng Song ${ }^{\mathrm{d}}$ \\ a Departamento de Física, Instituto Superior de Engenharia do Porto, Rua Dr. António Bernardino de Almeida, 431, 4200-072 Porto, Portugal \\ ${ }^{\mathrm{b}}$ Centro de Física, Universidade do Minho, 4700 Braga, Portugal \\ ${ }^{\mathrm{c}}$ Center of Optical Technology, Changchun Institute of Optics, fine Mechanics and Physics of Chinese Academy of Science, \\ PO Box 1024, 16\# East Nanhu Road, Changchun 130033, China \\ ${ }^{\mathrm{d}}$ Thin Film Centre, University of Paisley, High St, Paisley, PA1 2BE, Scotland
}

Available online 13 July 2007

\begin{abstract}
ITO films were deposited onto glass substrates by ion beam assisted deposition method. The oxygen ions were produced using a Kaufman ion source. The oxygen flow was varied from 20 until $60 \mathrm{sccm}$ and the effect of the oxygen flow on properties of ITO films has been studied. The structural properties of the film were characterized by X-ray diffraction and atomic force microscopy. The optical properties were characterized by optical transmission measurements and the optical constants (refractive index $\mathrm{n}$ and extinction coefficient $\mathrm{k}$ ) and film thickness have been obtained by fitting the transmittance using a semi-quantum model. The electrical properties were characterized by Hall effect measurements. It has been found that the ITO film with low electrical resistivity and high transmittance can be obtained with 40 sccm oxygen flow (the working pressure is about $2.3 \times 10^{-2} \mathrm{~Pa}$ at this oxygen flow).
\end{abstract}

(C) 2007 Elsevier B.V. All rights reserved.

Keywords: ITO; Thin Film; Ion beam assisted deposition; IR reflectance; optical and electrical properties

\section{Introduction}

The simultaneous occurrence of high optical transmission (over $80 \%$ ) in the visible region and low electrical resistivity (less than $10^{-3} \Omega \mathrm{cm}$ ) is not possible in an intrinsic stoichiometric material. The only way to obtain good transparent conductors is to create electron degeneracy in a wide band gap (over $3 \mathrm{eV}$ ) oxide by controllably introducing non-stoichiometry and/or appropriate dopants [1]. Indium tin oxide (ITO) is a tin doped $\operatorname{In}_{2} \mathrm{O}_{3}$ based ntype wide band gap degenerate semiconductor. ITO thin films combine high optical transmittance and low electrical resistivity and are widely used as transparent electrodes for various optoelectronic applications, such as solar cell, flat panel displays, organic light emitting diodes, electrochromic device and antistatic coatings [2-6]. Many techniques have been used to deposit ITO films, such as evaporation [7], sputtering [8-10], chemical vapour

\footnotetext{
* Corresponding author. Departamento de Física, Instituto Superior de Engenharia do Porto, Rua Dr. António Bernardino de Almeida, 431, 4200072 Porto, Portugal. Fax: +351228321159 .

E-mail address: 1jm@isep.ipp.pt (L.-J. Meng).
}

deposition [11], sol-gel [12], spray pyrolysis [13] and ion beam assisted deposition [14-17]. In general, either high substrate temperature (over $300^{\circ} \mathrm{C}$ ), or post-deposition annealing is needed for getting high quality ITO films. However, room temperature deposition of ITO films is desired for preparation of organic luminescent devices because of the low thermal stability of the organic materials. It is still a significant challenge to prepare high quality ITO films without heating the substrate and any postdeposition annealing process. Ion beam assisted deposition employs a separate ion source to direct a beam of ions at the growing film during deposition. Ion bombardments at substrate can supply sufficient energies to increase adatom mobility and chemical activity. Therefore, it has been considered as a technique to deposit ITO films at room temperature. In this work, we studied the effect of the oxygen flow on the structural, optical and electrical properties of the ITO films prepared by ion beam assisted deposition.

\section{Experimental details}

ITO films were deposited onto the commercial $\mathrm{k} 9$ glass substrates at room temperature by ion beam assisted deposition 
Table 1

Deposition conditions and physical properties of the ITO films deposited at different oxygen flows

\begin{tabular}{|c|c|c|c|c|c|}
\hline Sample & $\mathrm{O} 0$ & $\mathrm{O} 1$ & $\mathrm{O} 2$ & $\mathrm{O} 3$ & $\mathrm{O} 4$ \\
\hline Oxygen flow (sccm) & 60 & 50 & 40 & 30 & 20 \\
\hline Working pressure $\left(\times 10^{-2} \mathrm{~Pa}\right)$ & 3.4 & 2.9 & 2.3 & 1.8 & 1.4 \\
\hline Thickness (nm) & 292 & 261 & 228 & 223 & 301 \\
\hline Sheet resistance $(\Omega /$ square $)$ & 243 & 135 & 94 & 101 & 101 \\
\hline Electrical resistivity $\left(\times 10^{-3} \Omega \mathrm{cm}\right)$ & 32 & 16 & 9.7 & 10 & 14 \\
\hline Carrier concentration $\left(\times 10^{20} \mathrm{~cm}^{-3}\right)$ & 0.37 & 0.50 & 0.80 & 0.86 & 1.40 \\
\hline Hall mobility $\left(\mathrm{cm}^{2} / \mathrm{VS}\right)$ & 23.7 & 35.0 & 36.6 & 32.0 & 14.6 \\
\hline $\begin{array}{l}\text { Surface rms roughness }(\mathrm{nm}) \\
\text { d-spacing between }(222) \text { planes }(\mathrm{nm})\end{array}$ & 2.4 & 1.4 & 0.3 & 1.2 & 1.7 \\
\hline$\left(\right.$ Standard value $\left.d_{0}=0.2923 \mathrm{~nm}\right)$ & 0.2968 & 0.2985 & 0.2973 & 0.2969 & 0.2956 \\
\hline Grain size along (222) direction (nm) & 12 & 10 & 6 & 4 & 3 \\
\hline Calculated reflectance & $19 \%$ & $34 \%$ & $45 \%$ & $42 \%$ & $42 \%$ \\
\hline Measured reflectance at $12,000 \mathrm{~nm}$ & $31 \%$ & $39 \%$ & $45 \%$ & $46 \%$ & $47 \%$ \\
\hline
\end{tabular}

technique using a vacuum coater equipped with two electron beam guns (only one of them was used in this work) and a Kaufman ion source. ITO powder pellet with a composition of $90 \mathrm{wt} . \% \mathrm{In}_{2} \mathrm{O}_{3}$ and 10 wt. $\% \mathrm{SnO}_{2}$ was used as the evaporation source material. A $120 \mathrm{~mm}$ diameter Kaufman ion source was used to generate oxygen ion beam. The oxygen gas flow was controlled by a mass flow controller. The deposition rate and the film thickness were monitored and controlled by a quartz crystal sensor which has been linked to e-beam power supply for automatic controlling. The nominal deposition rate and the thickness were preset at $0.15 \mathrm{~nm} / \mathrm{s}$ and $200 \mathrm{~nm}$, respectively. The substrate holder was rotated at a speed of 0.3 rounds/s. The angle between the incident oxygen ion beam and the normal of the substrate holder was fixed at $45^{\circ}$. Before the deposition, the chamber was evacuated until a pressure of $1 \times 10^{-3} \mathrm{~Pa}$. After that, the oxygen gas was introduced into the chamber. The oxygen flow was set to be $60,50,40,30$ and $20 \mathrm{sccm}$, respectively. And the dynamic pressure in the chamber was about $3.4 \times 10^{-2}, 2.9 \times 10^{-2}, 2.3 \times 10^{-2}, 1.8 \times 10^{-2}$ and $1.4 \times 10^{-2} \mathrm{~Pa}$, respectively. The film thickness indicated in the Table 1 was obtained by fitting the transmittance spectra. During all depositions, the ion beam current, the accelerating voltage and the screen voltage were kept constants at $100 \mathrm{~mA}, 250 \mathrm{~V}$ and $500 \mathrm{~V}$, respectively.

The optical transmittance spectra of the films were recorded by Perkin-Elmer Lambda 900 UV/VIS/NIR spectrometer and the infrared reflectance was measured by Perkin-Elmer Spectrum GX at angle of incidence of $60^{\circ}$ related to the substrate normal. Atomic force microscopy (AFM) measurements were made using equipment from Digital Instruments Veeco Metrology Group. The AFM measurements have been done by tapping mode using $\mathrm{SiN}$ tip with $1.5 \mathrm{~Hz}$ scanning rate. The X-ray diffraction was done by SHIMADZU XRD-6000 performed between the $2 \theta$ values of $20^{\circ}-70^{\circ}$ with a step of $0.05^{\circ} . \mathrm{Cu} \mathrm{K \alpha}$ radiation from an X-ray tube with normal focus was used. The Hall effect was measured using Lake Shore 665 with a $5 \mathrm{kG}$ magnetic field intensity at room temperature. All the experimental error depend on the respective measuring machines.

\section{Results and discussion}

The X-ray diffraction measurements revealed that the ITO films deposited at different oxygen flows show a polycrystalline
$\mathrm{In}_{2} \mathrm{O}_{3}$ structure [18] and have a preferred orientation along the (222) direction as shown in Fig. 1. The broad amorphous phase is from the glass substrate. It can be seen that the (222) peak intensity decreases and the peak width broadens as the oxygen flow is decreased. The structure of the deposited ITO film is not only related with the deposition techniques, but also the deposition conditions. For example, at low ion beam energy, only amorphous ITO film can be obtained [19]. By fitting the (222) X-ray diffraction peak, the distance between the (222) crystal planes has been calculated and the grain size along the (222) direction has been estimated using Scherrer formula [20]. The results are given in Table 1 and shown in Fig. 2. The arrow

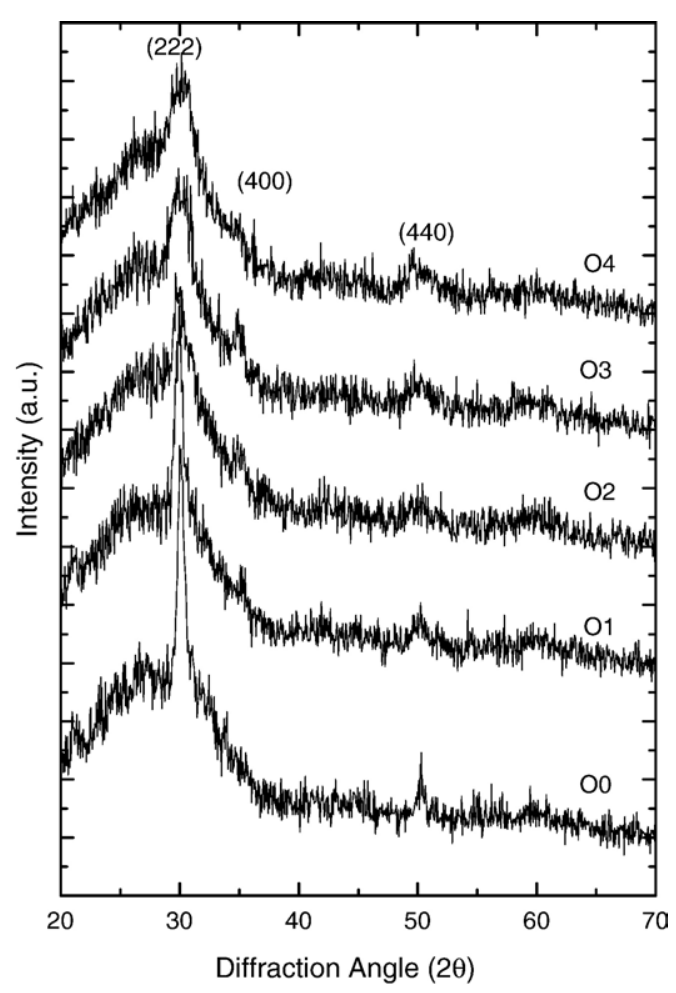

Fig. 1. XRD spectra for the ITO films deposited at different oxygen flows. (The oxygen flow is $60,50,40,30$ and $20 \mathrm{sccm}$ for sample O0, O1, O2, O3 and O4 respectively). 


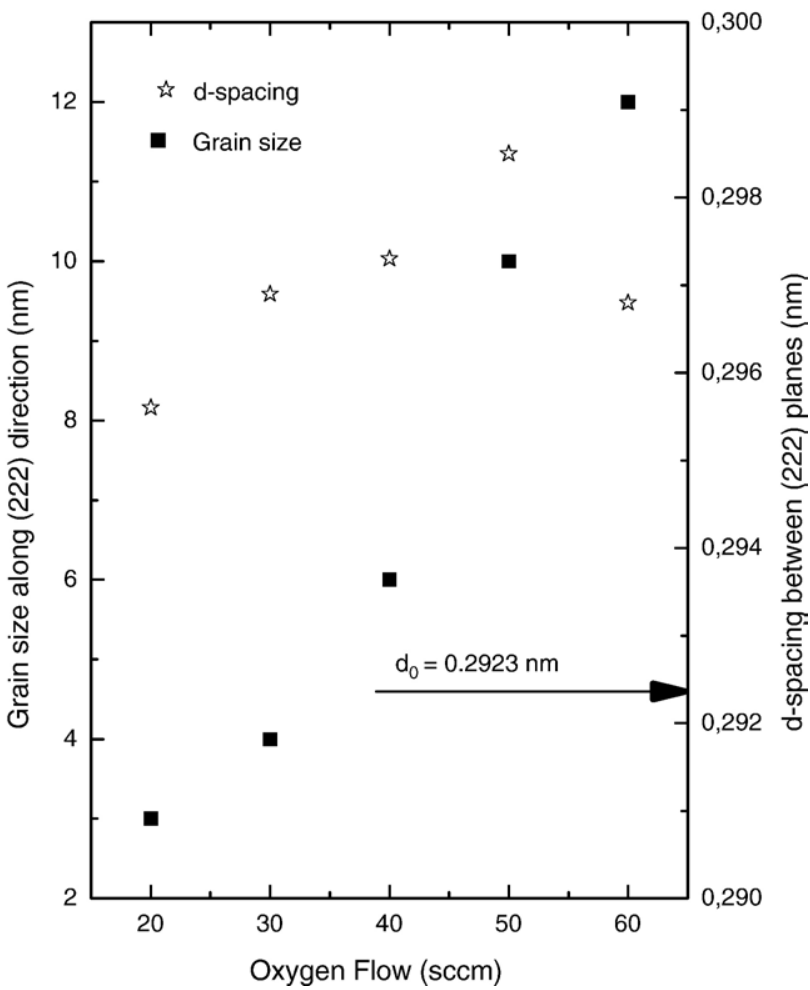

Fig. 2. The d-spacing between the (222) planes and grain size along (222) direction for ITO films deposited at different oxygen flows.

in the Fig. 2 indicates the standard value $d_{0}(0.2923 \mathrm{~nm})$. It shows that the $d$ values of all the films are larger than $d_{0}$ and the difference (between $d$ and $d_{0}$ ) increases as the oxygen flow is increased and reaches to maximum value when the oxygen flow is $50 \mathrm{sccm}$. That means that there are compressive stresses in all the films and the stress value has a maximum value at $50 \mathrm{sccm}$

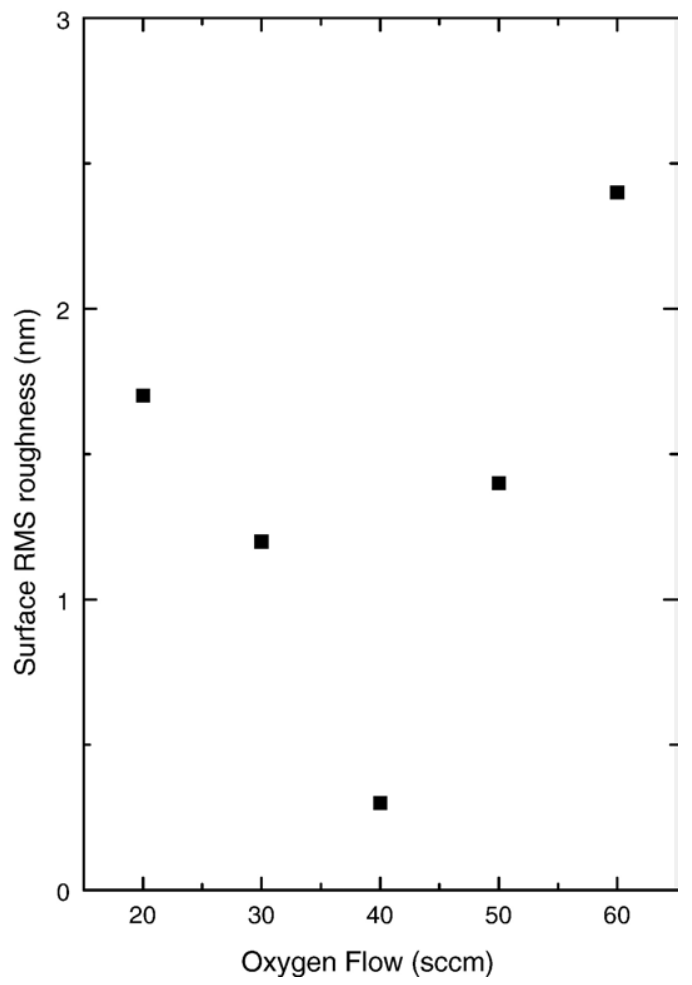

Fig. 4. Surface RMS roughness for ITO films deposited at different oxygen flows.

oxygen flow. The grain size along (222) direction increases gradually as the oxygen flow is increased. It means that the high oxygen flow is favourable to form big grains in the deposited ITO films.

The topography of the ITO films deposited at different oxygen flows has been evaluated by AFM measurements as
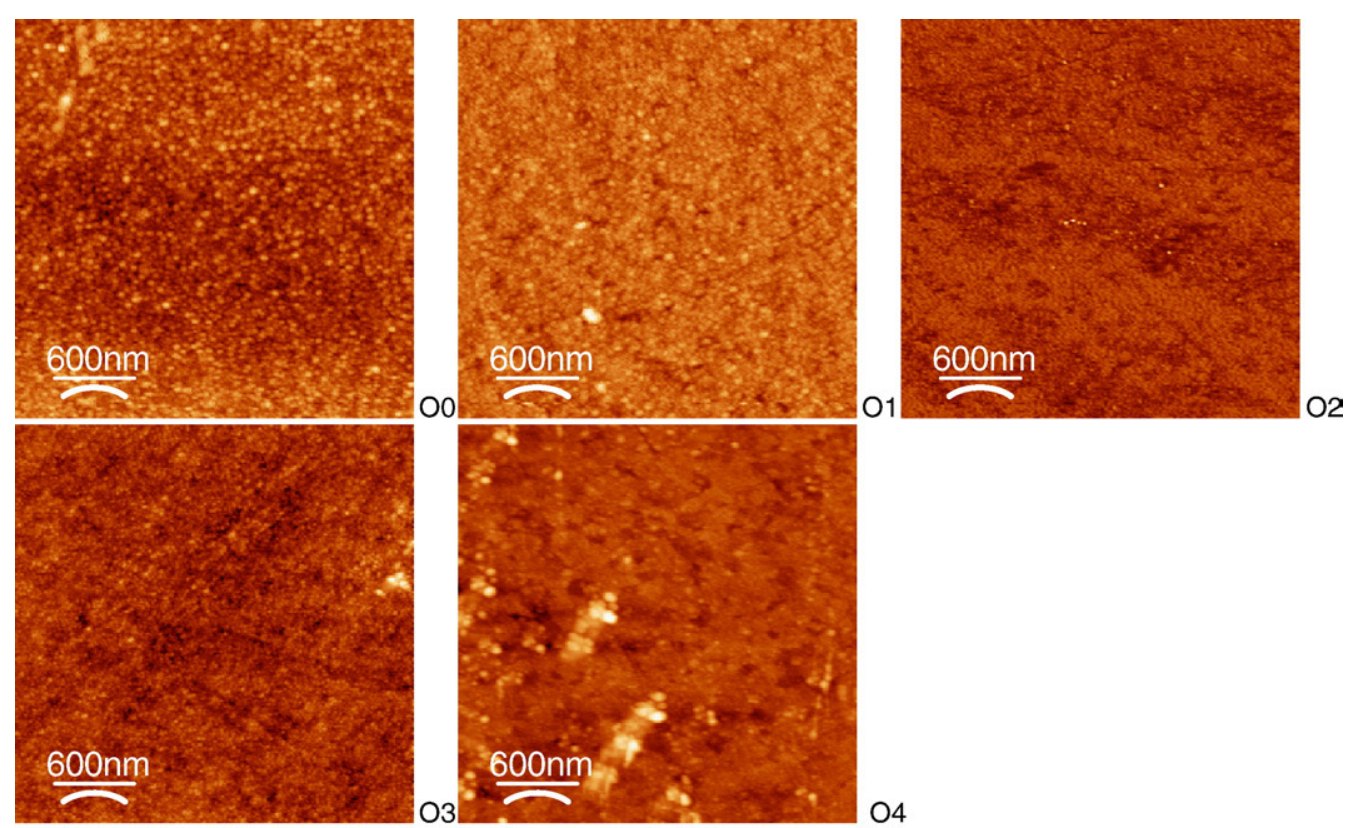

Fig. 3. AFM images $(3 \mu \mathrm{m} \times 3 \mu \mathrm{m})$ for the ITO films deposited at different oxygen flows. (The oxygen flow is $60,50,40,30$ and 20 sccm for sample O0, O1, O2, O3 and $\mathrm{O} 4$ respectively). 


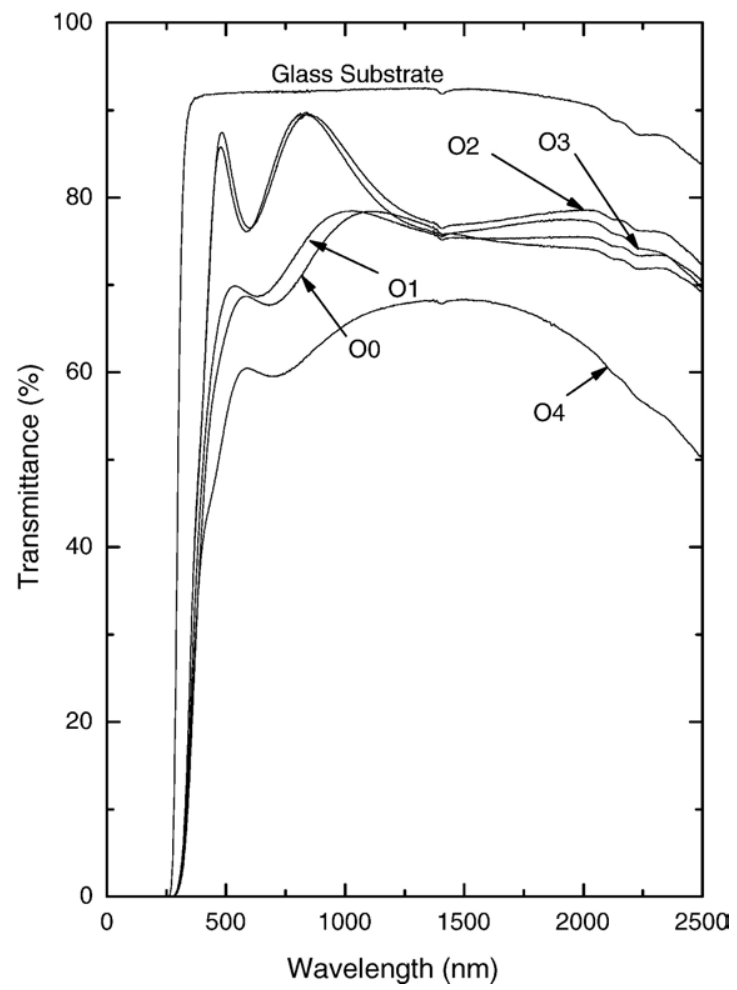

Fig. 5. Specular transmittance for the ITO films deposited at different oxygen flows. (The oxygen flow is $60,50,40,30$ and $20 \mathrm{sccm}$ for sample $\mathrm{O} 0, \mathrm{O} 1, \mathrm{O} 2$, $\mathrm{O} 3$ and $\mathrm{O} 4$ respectively).

shown in Fig. 3. The surface rms roughness values calculated from these images are given in Table 1 and shown in Fig. 4. The roughness decreases from $1.7 \mathrm{~nm}$ to $0.3 \mathrm{~nm}$ as the oxygen flow is increased from $20 \mathrm{sccm}$ to $40 \mathrm{sccm}$. When the oxygen flow is increased further, the roughness increases too.

The transmittance of the ITO films deposited at different oxygen flows are given in Fig. 5. The ITO film deposited at low oxygen flow $(20 \mathrm{sccm})$ gives a low transmittance in the visible and near infrared region. As the oxygen flow is increased (30 and $40 \mathrm{sccm}$ ), the transmittance of the ITO films increases too. However, when the oxygen flow is increased further (50 and $60 \mathrm{sccm}$ ), the transmittance in the visible region starts to decrease. When $\mathrm{In}_{2} \mathrm{O}_{3}$ and $\mathrm{SnO}_{2}$ mixed oxides pellet are evaporated, the oxygen will be lost and a metal-like, brownish and less transparent film of lower oxide will be formed. The low oxygen flow $(20 \mathrm{sccm})$ may not be enough to compensate the loss of the oxygen during the evaporation process and result in a low transmittance. The high oxygen flow (30 and $40 \mathrm{sccm}$ ) can compensate the loss of the oxygen during the evaporation

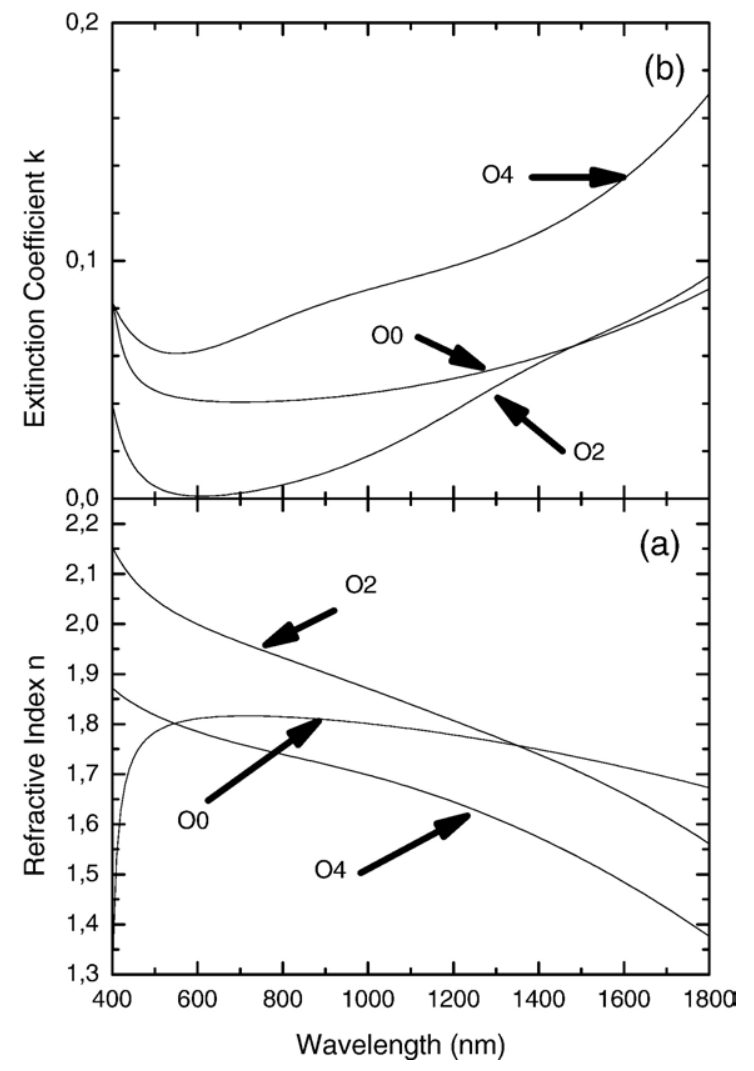

Fig. 6. Refractive index $\mathrm{n}$ (a) and extinction coefficient $\mathrm{k}$ (b) for the ITO films deposited at different oxygen flows. (The oxygen flow is 60,40 and $20 \mathrm{sccm}$ for sample $\mathrm{O} 0, \mathrm{O} 2$ and $\mathrm{O} 4$ respectively).

process and result in a high transmittance. When the oxygen flow is increased further (50 and $60 \mathrm{sccm}$ ), the surface roughness of the film increases as shown in Fig. 4. The rough surface will result in more scattering light and then a low transmittance. In addition, later it can be seen that the film prepared at high oxygen flow has a loose packing structure, it may also reduce the transmittance. It can be seen from Fig. 5 that there is a difference between the transmittance of the films prepared at low oxygen flows (less than $20 \mathrm{sccm}$ ) and the films prepared at high oxygen flows (more than $50 \mathrm{sccm}$ ), although they both have low transmittance values. For the film prepared at low oxygen flow, the transmittance is low both in the visible and near infrared region. For the film prepared at high oxygen flow, the transmittance only decreases in the visible region and still maintains a high value in the infrared region.

In order to get the optical constant of the ITO films, the transmittance (between $400 \mathrm{~nm}$ and $1800 \mathrm{~nm}$ ) have been fitted

\begin{tabular}{|c|c|c|c|c|c|c|c|c|c|c|c|c|}
\hline & $\varepsilon_{\infty}$ & $\omega_{\mathrm{p}}\left(\mathrm{cm}^{-1}\right)$ & $\gamma_{\mathrm{d}}\left(\mathrm{cm}^{-1}\right)$ & $\omega_{\mathrm{LO} 1}\left(\mathrm{~cm}^{-1}\right)$ & $\gamma_{\mathrm{LO} 1}\left(\mathrm{~cm}^{-1}\right)$ & $\omega_{\mathrm{TO} 1}\left(\mathrm{~cm}^{-1}\right)$ & $\gamma_{\mathrm{TO} 1}\left(\mathrm{~cm}^{-1}\right)$ & $\omega_{\mathrm{LO} 2}\left(\mathrm{~cm}^{-1}\right)$ & $\gamma_{\mathrm{LO} 2}\left(\mathrm{~cm}^{-1}\right)$ & $\omega_{\mathrm{TO} 2}\left(\mathrm{~cm}^{-1}\right)$ & $\gamma_{\mathrm{TO} 2}\left(\mathrm{~cm}^{-1}\right)$ & Discrepancy \\
\hline $\mathrm{O} 0$ & 3,56 & 5149 & 884 & 2123 & 23784 & 605 & 22507 & 25458 & 0,42 & 26106 & 237 & 0.005 \\
\hline $\mathrm{O} 1$ & 3,97 & 4665 & 10228 & 5874 & 4060 & 5942 & 3932 & 27101 & 26 & 28367 & 696 & 0.005 \\
\hline $\mathrm{O} 2$ & 3,33 & 7195 & 731 & 7200 & 5069 & 7132 & 5052 & 36809 & 9974 & 33651 & 7731 & 0.005 \\
\hline $\mathrm{O} 3$ & 3,41 & 7615 & 612 & 7645 & 6096 & 7543 & 6146 & 36798 & 9974 & 33795 & 8007 & 0.005 \\
\hline $\mathrm{O} 4$ & 2,65 & 7201 & 1039 & 12141 & 15037 & 11771 & 15040 & 46406 & 7882 & 41554 & 9149 & 0.005 \\
\hline
\end{tabular}


using semi-quantum model [21,22] combined with Drude model. The dielectric function can be described as follows:

$\varepsilon(\omega)=\varepsilon_{\infty} \prod_{j} \frac{\omega_{\mathrm{jLO}}^{2}-\omega^{2}-i \gamma_{\mathrm{jLO}} \omega}{\omega_{\mathrm{jTO}}^{2}-\omega^{2}-i \gamma_{\mathrm{jTO}} \omega}+\frac{\omega_{p}^{2}}{-\omega^{2}+i \gamma_{d} \omega}$

The first term is the semi-quantum model and it represents the dielectric function as a product of individual oscillator terms. For each term there are four parameters, where $\omega_{\mathrm{j} \text { то, }}$ $\gamma_{\mathrm{jTO}}, \omega_{\mathrm{jLO}}, \gamma_{\mathrm{jLO}}$ are the resonance frequencies and damping constants of the transverse and longitudinal optic modes, respectively. $\varepsilon_{\infty}$ is the "high-frequency" contribution to the dielectric function. The second term is the Drude model which is used to modify the free electron properties. Very good fits have been obtained for all the ITO films using these models. The fitting parameters are listed in Table 2.

After fitting, the refractive index and extinction coefficient can be obtained as shown in Fig. 6. It can be seen that the ITO film prepared at $40 \mathrm{sccm}$ oxygen flow shows a high refractive index and low extinction coefficient. ITO films prepared at both the low oxygen flow $(20 \mathrm{sccm})$ and high oxygen flow (higher than $50 \mathrm{sccm}$ ) have low refractive index and high extinction coefficient. The low refractive index means a loose packing structure and a low packing density. Therefore, both the ITO films prepared at low and high oxygen flows have a low packing density.

Fig. 7 gives the electrical properties of ITO films deposited at different oxygen flows. It can be seen that the electrical resistivity has a small decrease when the oxygen flow is

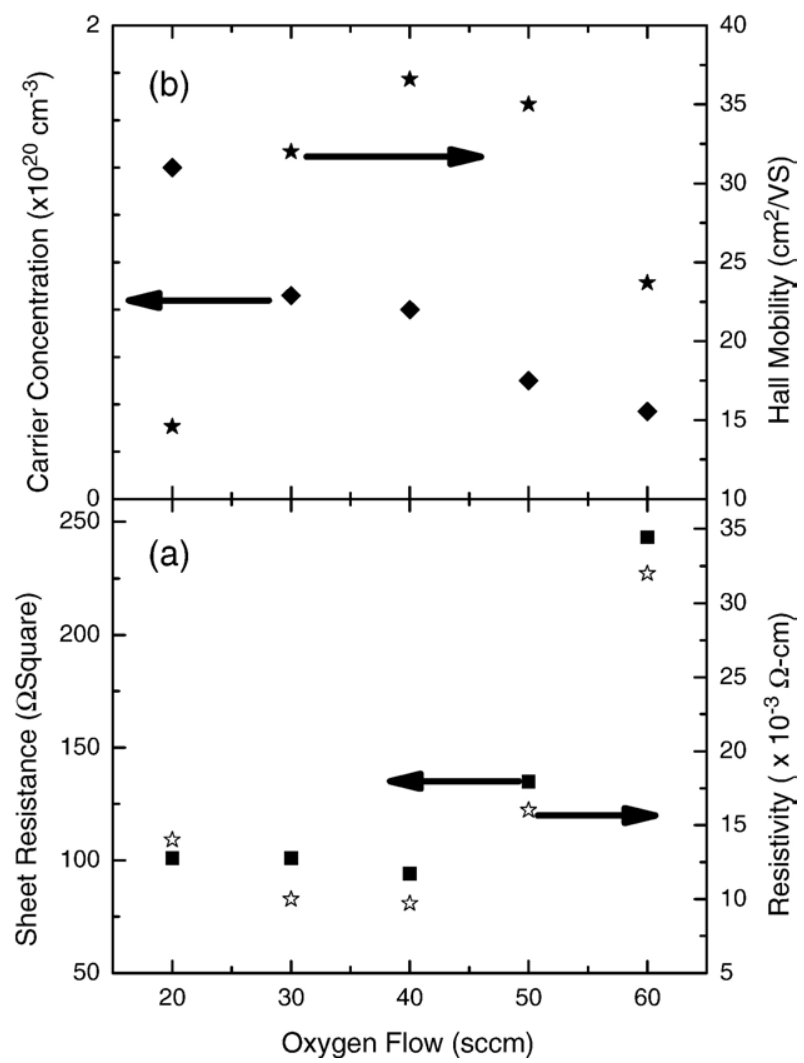

Fig. 7. The variation of sheet resistance and electrical resistivity (a) and carrier concentration and Hall mobility (b) with the oxygen flow.

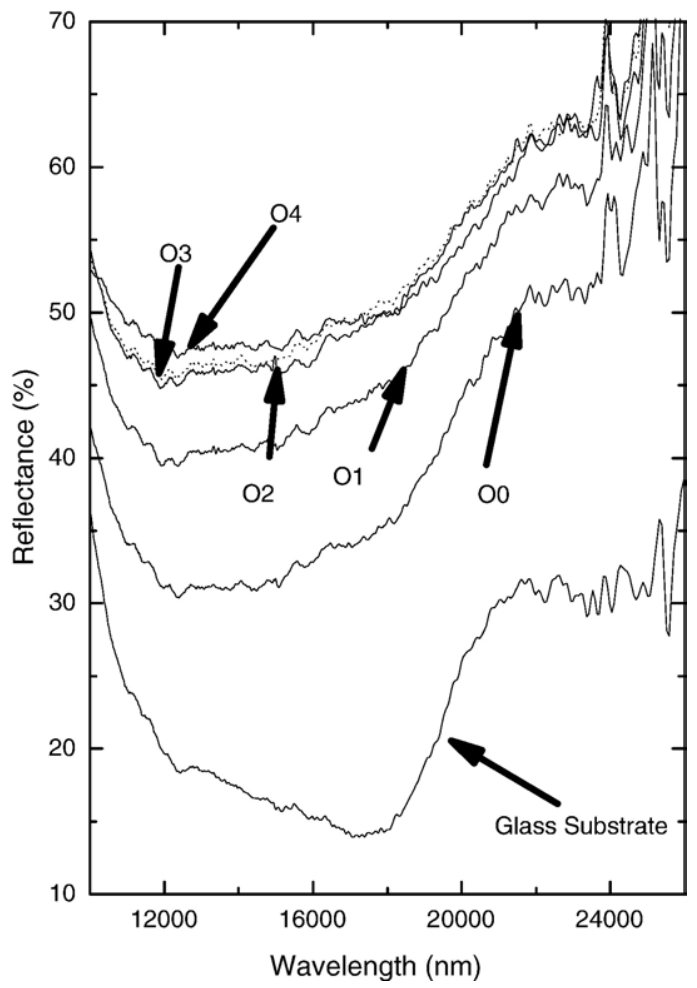

Fig. 8. IR reflectance for the ITO films prepared at different oxygen flows. (The oxygen flow is $60,50,40,30$ and 20 sccm for sample O0, O1, O2, O3 and $\mathrm{O} 4$ respectively).

increased from 20 to $40 \mathrm{sccm}$, after that, the electrical resistivity starts to increase with the increase of the oxygen flow. Hall effect measurements show (Fig. 8b) that the electron concentration decreases gradually as the oxygen flow is increased. However, the Hall mobility reach the maximum value at the $40 \mathrm{sccm}$ oxygen flow and result in a minimum value of the electrical resistivity. The one source of the electron in ITO film is from oxygen vacancies. As the oxygen flow is increased, the oxygen vacancies vanish and lead to a decrease of the electron concentration. It has been known that the surface roughness will influence the carrier mobility. Rough surface results in a decrease of the carrier mobility. From Fig. 4 it can be seen that the surface roughness of the ITO film deposited at $40 \mathrm{sccm}$ oxygen flow has the minimum value and results in high carrier mobility. In addition, the non-stoichiometric structure (films prepared at low and high oxygen flows) may also cause the low electron mobility. Usually, the grain size should have the effect on the carrier mobility because of the grain boundary scattering. As it can be seen from the Table 1 the sample prepared at $60 \mathrm{sccm}$ oxygen flow has the biggest grain size, if it is the main factor for the variation of the carrier mobility, the sample should have the highest mobility. But the highest mobility is obtained for samples prepared at $40 \mathrm{sccm}$ oxygen flow. Therefore, the grain size is not the main reason for the variation of the carrier mobility in this work.

Fig. 8 shows the reflectance FTIR spectra for ITO films prepared at different oxygen flows. The spectra shown were obtained for angles of incidence of $60^{\circ}$ related to the substrate normal. When the oxygen flow is decreased from 60 to $40 \mathrm{sccm}$, the reflectance has a clear increase. After that, even the oxygen flow is 
decreased further (from 40 to $20 \mathrm{sccm}$ ), the reflectance only shows a small increase. There is a very simple relation between the infrared reflection $\mathrm{R}$ and the sheet resistance $R_{\mathrm{W}}[12,23]$ :

$R_{\mathrm{IR}}=\left(1+2 \varepsilon_{0} c_{0} R_{\mathrm{W}}\right)^{-2}$

The reflectance of ITO films deposited at different oxygen flows has been calculated by this equation. The results are listed in Table 1. For comparing, the measured reflectances at 12,000 nm are also listed in the Table. It can be seen that only for the sample prepared at $40 \mathrm{sccm}$ oxygen flow, the calculated value is in good accordance with the measured value. It means this equation can only give the good result for the sample with low sheet resistance.

\section{Conclusions}

ITO thin films have been deposited onto glass substrates at room temperature by ion beam assisted deposition technique at different oxygen flows (20 to $60 \mathrm{sccm}$ ). The films show a polycrystalline ITO structure with a preferred orientation along (222) direction. There is a compressive stress in all deposited ITO films. The grain size along (222) direction increases with oxygen flow. The surface rms roughness decreases as the oxygen flow is increased from 20 to $40 \mathrm{sccm}$, after that, the roughness increases again with oxygen flow. The ITO films prepared at 40 and $50 \mathrm{sccm}$ oxygen flows have good optical transmission. The ITO films prepared at oxygen flow lower and higher than these values have poor optical transmission. The transmittance spectra have been fitted using the semi-quantum model combined with the Drude model. The refractive index and extinction coefficient have been obtained after fitting the transmittance spectra. The ITO film prepared at $40 \mathrm{sccm}$ oxygen flow shows a high refractive index and a low extinction coefficient. The electron concentration decreases gradually as the oxygen flow is increased. The electron mobility increases with oxygen flow and reach to maximum value at $40 \mathrm{sccm}$ oxygen flow, after that, it decreases again with the oxygen flow. This variation results in a low electrical resistivity for ITO film deposited at $40 \mathrm{sccm}$ oxygen flow. The infrared reflectance of ITO film shows a very clear increase when the oxygen flow is decreased from $60 \mathrm{sccm}$ to $40 \mathrm{sccm}$. And a small increase when the oxygen flow is decreased from 40 to $20 \mathrm{sccm}$. The infrared reflectance can be related to the sheet resistance, the calculated reflectance is in good accordance with the measured reflectance for ITO films with low sheet resistance.

\section{Acknowledgments}

This work was carried out in the Centre of Optical Technology, Changchun Institute of Optics, fine Mechanics and Physics of Chinese Academy of Sciences during Li-Jian Meng's visit to the centre as a senior visiting researcher. Li-Jian Meng is thankful to the Fundação para a Ciência e a Tecanologia (Portugal) for providing a fellowship (SFRH-BSAB-514).

\section{References}

[1] K.L. Chopra, S. Major, D.K. Pandya, Thin Solid Films 102 (1983) 1.

[2] M. Terai, D. Kumaki, T. Yasuda, K. Fujita, T. Tsutsui, Curr. Appl. Phys. 5 (2005) 341 .

[3] G.G. Gao, L. Xu, W.J. Wang, W.J. An, Y.F. Qiu, Z.Q. Wang, E.B. Wang, J. Phys. Chem., B 109 (2005) 8948.

[4] B.H. Lee, I.G. Kim, S.W. Cho, S.H. Lee, Thin Solid Films 302 (1997) 25.

[5] A. Gadisa, M. Svensson, M.R. Andersson, O. Inganas, Appl. Phys. Lett. 84 (2004) 1609.

[6] H.P. Lobl, M. Huppertz, D. Mergel, Surf. Coat. Technol. 82 (1996) 90.

[7] J. Ma, D.H. Zhang, J.Q. Zhao, C.Y. Tan, T.L. Yang, H.L. Ma, Appl. Surf. Sci. 151 (1999) 239.

[8] L.J. Meng, A. Maçarico, R. Martins, Vacuum 46 (1995) 673.

[9] L.J. Meng, M.P. dos Santos, Thin Solid Films 303 (1997) 151.

[10] L.J. Meng, E. Crossan, A. Voronov, F. Placido, Thin Solid Films 422 (2002) 80 .

[11] K. Maki, N. Komiya, A. Suzuki, Thin Solid Films 445 (2003) 224.

[12] P.K. Biswas, A. De, N.C. Pramanik, P.K. Chakraborty, K. Ortner, V. Hock, S. Korder, Mater. Lett. 57 (2003) 2326.

[13] H. El Rhaleb, E. Benamar, M. Rami, J.P. Roger, A. Hakam, A. Ennaoui, Appl. Surf. Sci. 201 (2002) 138.

[14] D. Kim, S. Kim, Thin Solid Films 408 (2002) 218.

[15] C. Liu, T. Mihara, T. Matsutani, T. Asanuma, M. Kiuchi, Solid State Commun. 126 (2003) 509.

[16] P.J. Martin, R.P. Neterfield, D.R. McKenzie, Thin Solid Films 137 (1986) 207.

[17] M. Gilo, R. Dahan, N. Croitoru, Opt. Eng. 38 (1999) 953.

[18] Powder Diffraction File, Joint Committee on Powder Diffraction Standards, 1967 (ASTM, Philadelphia, PA, 1967) Card 6-0416.

[19] C. Liu, T. Matsutani, T. Asanuma, M. Kiuchi, Nucl. Instrum. Methods Phys. Res., B Beam Interact. Mater. Atoms 206 (2003) 348.

[20] D. Cullity, Elements of X-ray Diffraction, 2nd ed.Addison-wesley, Reading, MA, 1978.

[21] F. Gervais, B. Piriou, Phys. Rev., B 11 (1975) 3944.

[22] M. Schubert, T.E. Tiwald, C.M. Herzinger, Phys. Rev., B 61 (2000) 8187.

[23] G. Frank, E. Kauer, H. Kostlin, Thin Solid Films 77 (1981) 107. 